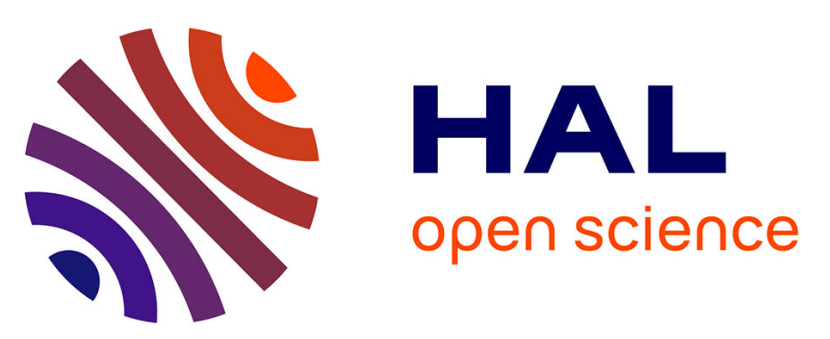

\title{
LA DISSOCIATION DES DISLOCATIONS DANS LE GERMANIUM OBSERVÉE PAR MICROSCOPIE ÉLECTRONIQUE A HAUTE RÉSOLUTION
}

\author{
J. Desseaux, A. Bourret
}

\section{- To cite this version:}

J. Desseaux, A. Bourret. LA DISSOCIATION DES DISLOCATIONS DANS LE GERMANIUM OBSERVÉE PAR MICROSCOPIE ÉLECTRONIQUE A HAUTE RÉSOLUTION. Journal de Physique Colloques, 1978, 39 (C2), pp.C2-116-C2-117. 10.1051/jphyscol:1978218 . jpa-00217364

HAL Id: jpa-00217364

https://hal.science/jpa-00217364

Submitted on 1 Jan 1978

HAL is a multi-disciplinary open access archive for the deposit and dissemination of scientific research documents, whether they are published or not. The documents may come from teaching and research institutions in France or abroad, or from public or private research centers.
L'archive ouverte pluridisciplinaire HAL, est destinée au dépôt et à la diffusion de documents scientifiques de niveau recherche, publiés ou non, émanant des établissements d'enseignement et de recherche français ou étrangers, des laboratoires publics ou privés. 


\title{
LA DISSOCIATION DES DISLOCATIONS DANS LE GERMANIUM OBSERVÉE PAR MICROSCOPIE ÉLECTRONIQUE A HAUTE RÉSOLUTION
}

\author{
J. DESSEAUX et A. BOURRET \\ Centre d'Etudes Nucléaires de Grenoble, Département de Recherche Fondamentale, \\ Section de Physique du Solide, 85 X, 38041 Grenoble Cedex, France
}

\begin{abstract}
Résumé. - La microscopie électronique à haute résolution permet de déterminer la largeur de dissociation des dislocations dans le germanium à une distance atomique près (résolution des plans atomiques). On trouve des dislocations dissociées ou non selon leur vecteur de Burgers.
\end{abstract}

Abstract. - High resolution electron microscopy shows the dissociation width of dislocations in Ge within one atomic distance (resolution of atomic planes). Some dislocations are found dissociated, but some others are not.

Les dislocations dans les semiconducteurs présentent une faible dissociation. L'observation en particulier de nœuds faiblement dissociés par Aerts et al. [1], bien que controversée, l'avait mise en évidence. Ray et Cockayne [2] devaient confirmer de manière non ambiguë la présence d'une dissociation grâce à la technique des faisceaux faibles en microscopie électronique. Ces auteurs montrèrent également que l'énergie de faute intrinsèque était supérieure à l'énergie de faute extrinsèque contrairement à ce qui a lieu dans les métaux. Cependant les échantillons observés étaient particulièrement impurs. Par ailleurs les observations à haute résolution des plans atomiques (111) dans le germanium au voisinage d'une dislocation ne montraient pas de dissociation [3] : cependant dans ce cas bien que les dislocations observées soient supposées être à $60^{\circ}$ aucune détermination du vecteur de Burgers par les méthodes classiques ne devait être faite. Nous avons donc repris ces expériences pour tenter de lever ces contradictions. De plus, nous avons utilisé pour observer les dislocations une technique à très haute résolution permettant de visualiser directement les plans atomiques [4] et donc d'obtenir des largeurs de dissociation avec une grande précision.

1. Méthodes expérimentales. - Nous avons utilisé des bicristaux présentant un sous-joint de pure flexion autour de [110]. Le plan du joint est voisin de (110). L'angle de joint est compris entre $0,5^{\circ}$ et $3^{\circ}$.

2. Résultats. - Le sous-joint se présente sous forme de dislocations isolées alignées en moyenne dans une direction (002). Dans les parties rectilignes du joint les dislocations sont purement coin de vecteur de Burgers $1 / 2(1 \overline{1} 0)$ comme on doit s'y attendre pour un joint de flexion pure. Cependant les parties rectilignes sont séparées par des décrochements formés d'un alignement dans une direction voisine de (111) ou (111) de dislocations étrangères : la détermination du vecteur de Burgers de ces dislocations montre qu'ils sont alternativement du type $1 / 2(011)$ et $1 / 2$ (101); ces dislocations sont donc à $60^{\circ}$.

Le cœur des dislocations purement coin du joint de flexion (Fig. 1) est très peu étendu : il s'agit en effet d'une dislocation sessile dont la dissociation nécessiterait trois partielles dans deux plans (111).

Le cour des dislocations à $60^{\circ}$ étrangères est au contraire dissocié dans le plan de glissement de la dislocation. La figure 2 montre par exemple une dislocation à faute intrinsèque. Les déplacements loin du cœur calculés par la théorie élastique sont en accord à $0,5 \AA$ près avec les emplacements sur l'image. Cette méthode de superposition image-points calculés est utilisée pour rechercher la distance exacte, $d=30 \pm 3 \AA$, entre les partielles avec une précision d'une distance atomique. Ceci permet de déduire l'énergie de faute intrinsèque $\gamma_{1}=100 \pm 10 \mathrm{ergs} / \mathrm{cm}^{2}$. Nous n'avons pas pu mettre en évidence de faute

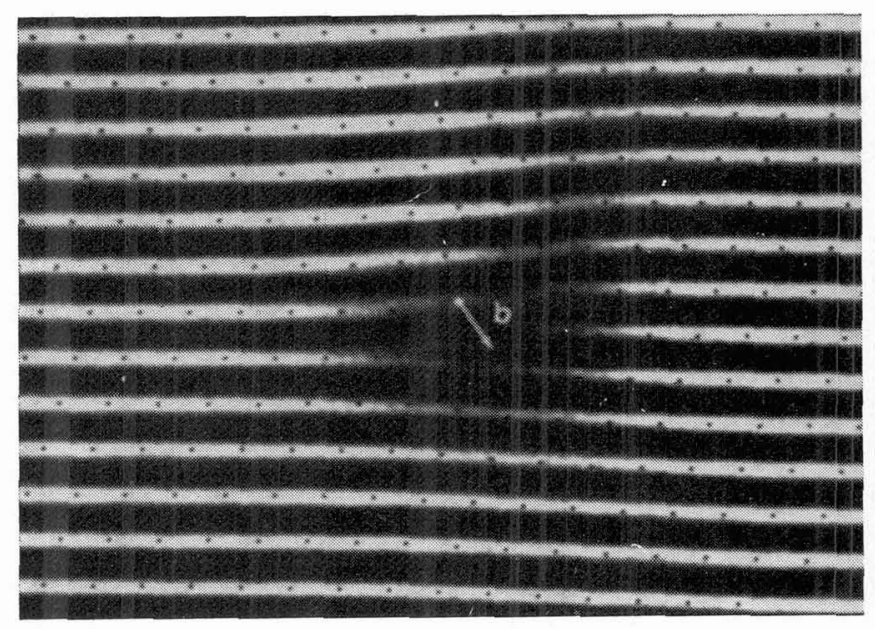

FIG. 1. - Coeur d'une dislocation sessile purement coin. [110] $b=1 / 2[1 \overline{1} 0]$ dans le germanium. 


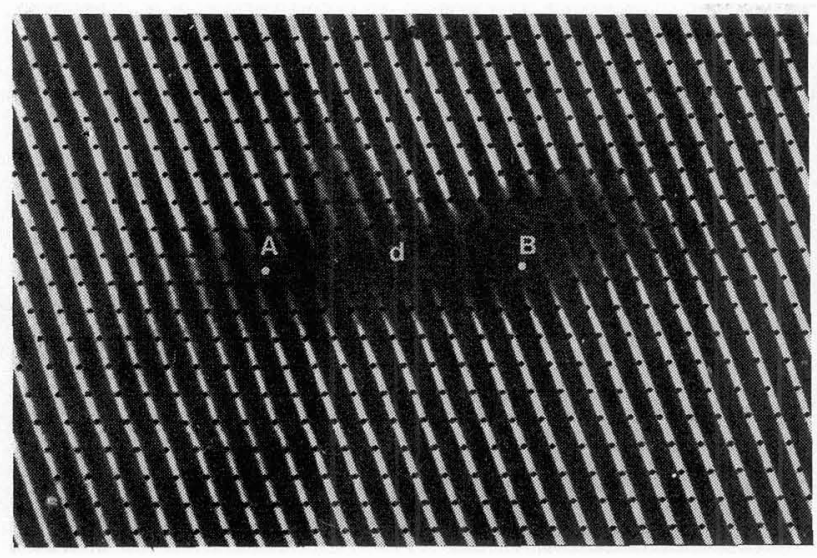

FIG. 2. - Cœur d'une dislocation à $60^{\circ}[110] b=1 / 2[011]$ à faute intrinsèque, les points noirs indiquent les positions atomiques calculées selon la théorie élastique. extrinsèque. L'énergie de faute intrinsèque ainsi mesurée est plus élevée que la valeur obtenue par Ray et Cockayne; ceci tient probablement à une meilleure pureté de nos échantillons. De récentes mesures par faisceau faible [5] se rapprochent d'ailleurs de nos résultats.

3. Conclusion. - Ce travail confirme la présence simultanée de dislocations dissociées et non dissociées. Les dislocations non dissociées constituent cependant le cas particulier puisqu'il s'agit de dislocations sessiles (le plan de glissement n'est pas un plan 111). Les largeurs de dissociation mesurées sont nettement plus faibles que les valeurs publiées jusqu'à maintenant.

\section{Bibliographie}

[1] Aerts, E., Delavignette, P., Amelinckx, S., J. Appl. Phys. 33 (1962) 3078.

[2] RAY, I. L.F., COCKAYNe, D. J. H., Proc. R. Soc. A 325 (1973) 534.

[3] Phillips, V. A., Wagner, R., J. Appl. Phys. 44 (1973) 4252.

[4] Desseaux, J., Renault, A., Bourret, A., Phil. Mag. 35 (1977) 357.

[5] Packeiser, G., HaAsen, P., Phil. Mag. 35 (1977) 821. 